\title{
LYON, FERGUS - GUIDO MÖLLERING - MARK N. K. SAUNDERS (EDS.) (2012), HANDBOOK OF RESEARCH METHODS ON TRUST. CHELTENHAM, UNITED KINGDOM, EDWARD ELGAR
}

ZSANNA NYÍRÖ1

Trust is a fundamental social phenomenon; however, both its conceptualization and its measurement present serious challenges to researchers. This is because trust is a multi-faceted, complex, elusive and culturally-determined phenomenon. Furthermore, researchers have to take into account the fact that trust is a particularly sensitive subject.

The vast majority of research on trust has used surveys and experimental methods, although both solutions have their limitations. Questionnaires do not allow for the deeper exploration of the topic, while experiments are only able to capture the behavioral components of trust. This is where lies the importance of the Handbook of Research Methods on Trust, edited by Fergus Lyon, Guido Möllering and Mark N. K. Saunders. The book presents a broad array of innovative qualitative and quantitative (and mixed) methods which are suitable for measuring trust. This is also the main message of the book: a great variety of methods are needed to examine such a multi-faceted phenomena.

This review starts with a summary of the book, followed by an analysis of its strengths and weaknesses. The handbook is a collection of articles related to the empirical research methods which exist for investigating trust. The book is divided into three sections. Each of them reflects a different part of trust research. The first section of the book focuses on conceptual issues and empirical concerns related to them. The second section presents a wide range of qualitative techniques for researching trust, while the third discusses quantitative methods. Some of the studies (e.g. Chapter 11, Chapter 12) use both qualitative and quantitative methods.

1 Zsanna Nyíró is a Ph.D. student at the Corvinus University of Budapest, e-mail: nyzsanna@ gmail.com 
The introductory chapter summarizes the topics of the book and identifies six themes of trust research: antecedents of trust; processes of building trust; the context shaping trust-building; decision-making processes in trust; implications and uses of trust; lack of trust, distrust, mistrust and repair. Furthermore, this chapter offers a brief summary of the qualitative and quantitative research methods into trust. An important part of this chapter is the identification of the methodological challenges of research into trust. The authors emphasize five key difficulties in trust research: the dynamic processes of trust (the challenge of capturing the temporal element of trust); researching tacit or less visible forms of trust; conceptualizing and describing trust in different cultures; the role of researchers in shaping the trust situation they are researching, and ultimately, the research ethics of trust. Finally, the chapter introduces future directions for trust research.

Chapters 2-16 cover a broad array of qualitative methods. Chapter 2 focuses on investigating the antecedents (cognitive and social factors) of trust judgements by using autobiographical narratives and semi-structured interviews (instead of the more commonly-used laboratory studies). Chapter 3 discusses the conceptualization and operationalization of trust beliefs and behaviours and reveals two challenges: the measurement of trust and distrust as separate concepts, and the measurement of trust development, decline and repair over time. Chapter 4 introduces an examination of the building and breaking of trust by using an agent-based simulation. The next three conceptual chapters focus on the outcomes of trust at different levels. Chapter 5 provides the micro-level by researching trust in entrepreneurial activities in different cultures using mixed methods - survey, fieldwork and expert interviews. The conclusion suggests the need to apply culturally-sensitive approaches because of the context-dependent nature of trust. Chapter 6 examines the meso-level by studying inter-organizational relationships. The chapter concerns the investigation of the dynamic relationship between trust and social capital and states that a suitable method for assessing changes in trust and social capital is still missing. Chapter 7 discusses the macro-level by introducing the critiques and defence of the standard trust-related questions which are used to compare levels of trust between countries. Chapter 8 highlights the fact that the trust between the researcher and the research participant is important because of the sensitive nature of the topic. The relationship between researcher and the researched influences both the research results (validity issues) and access to informants (sampling issues). The chapter also details the experiences of a fieldwork conducted in Ghana. Chapter 9 presents an example of qualitative community research into trust; a rarely-used method in this field. Chapter 10 discusses the experiences of applying a cross-cultural case study in trust 
research. Chapter 11 again highlights the importance of rapport and trust between the researcher and the researched due to the sensitive nature of the topic. Furthermore, it is also emphasizes that the researcher has to ensure that participants are not sensitized to the focus of the research. The chapter suggests applying a new and rarely-used kind of method: card sorting combined with an in-depth interview in order to inform but not sensitize participants to the topic. The author also shares his own experiences related to the application of this method in trust research. Chapter 12 presents the use of the board game method in cross-cultural settings in trust research, which can be used by participants to conceptualize, operationalize and rank values (in this case values related to trustworthiness). By using this method, both qualitative data (definitions, operationalization, and reasoning about rank order) and quantitative data (ranking places) can simultaneously be collected, and the interpretation of ranks and rank orders can be compared between cultures. Chapter 13 also offers a new and seldom-applied method: the repertory grid technique, whose use is described with reference to the author's own research. The essence of repertory grid-based interviews is that the researcher provides a list of elements considered important to the participant with regards to the research topic and then the interviewee attaches meaning to these elements. After this, three elements are picked up at random by the researcher and the participant is asked if - in relation to trust - these elements are thought of as similar or not. In this way dimensions may be constructed by the interviewee. Finally, the participant is asked to rank each element using the dimensions they have created. This final step allows for statistical analysis of the results. Chapter 14 presents another example of the usage of the repertory grid, but this study combines the technique with narratives. Chapter 15 provides a hermeneutic analysis into trust. Chapter 16 focuses on the critical incident technique in trust research. The essence of this method is that participants are asked to report incidents in their life where creating, strengthening or destroying trust played a role. The authors also highlight their personal experiences related to the usage of this method and its advantages and disadvantages.

The next chapters (17-23) describe quantitative methods. Chapter 17 provides a thorough overview of survey-based measures of trust in an organizational context. The chapter reveals the limitations of trust measurement in the organizational sciences. The author identifies the main challenges of constructing a valid, reliable and multidimensional measure. The chapter offers some principles for constructing an appropriate measure and presents the solution (a Behavioural Trust Inventory). Chapter 18 presents a model (called the Actor-Partner Independence Model - APIM) which 
facilitates the understanding of trust as a dyadic trustor-trustee phenomenon where trust in one partner affects the trust of the other party. Chapter 19 offers an analytical approach to the study of trust in an embedded setting. The authors introduce a few hypotheses related to different social mechanisms and then present the advantages and disadvantages of three different methods (vignette experiments, laboratory experiments, and survey) to test them. Chapter 20 examines the potential for using quantitative decision analysis techniques in measuring trust decisions. Chapter 21 focuses on the role of using diary methods in trust research. This technique is based on an analysis of the participants' detailed descriptions about events and experiences in their lives. Chapter 22 deals with the use of indirect (implicit) measures of trust. These measures are based on the automatic activation of attitudes in order to minimise response biases. The last chapter (23) of the section focuses on analysing social signals in speech (e.g. emphasis and the activity level of speaking) and its significance in trust research.

The concluding chapter (24) evaluates the current practices of trust research on the basis of three features: accuracy, generality, and simplicity. The main message of the chapter is that trust researchers should become a community instead of remaining isolated in order to develop empirical and theoretical research about trust.

The handbook provides a broad array of qualitative and quantitative methods which are suitable for examining the complex and multi-level phenomenon that is trust. A wide range of methods - among them both the new and rarely-used - is presented; therefore the book is not only of interest to those who are seeking to investigate trust, but also to those who are interested in methodological issues or examining other multi-faceted phenomena similar to trust.

From what has already been said it is obvious that the structure of the book is thematic (conceptual issues, qualitative research, and quantitative research). However, the handbook does not completely follow this format. For example, Part I. (Conceptual issues) also contains empirical work (Chapter 2) and a study on methodological issues (Chapter 7). What is more important is that several studies used mixed methods (e.g. Chapter 11-14). The editors of the book also highlight the significance of using mixed methods in trust research: "...we acknowledge that trust research can and does mix both, either through drawing on different methods to examine different aspects sequentially, or by explicitly developing methods that use both qualitative and quantitative methods concurrently" (Lyon et al. 2012, p. 7.) Therefore it is not clear why the editors insisted on the (traditional) format of dividing the presentation of the research into qualitative and quantitative methods. 
In terms of the content, the main deficiency of the book is its lack of a summary overview of conceptual issues. Since the book discusses many types of trust, a chapter on this topic would have been useful to readers. It would have been also useful to summarize conceptual difficulties at the beginning of the book.

One of the aims of the book was to present a broad array of methods. Correspondingly, chapters are a little brief (about ten pages), so some detail about the studies is lacking. Also for the abovementioned reason, only a few studies deal with surveys - the most commonly-used technique in trust research.

As far as I can see, the book also lacks research about trust in institutions. Currently, trust in institutions is a very important area of trust research.

A major advantage of the studies in this book is that the authors of the chapters always conceptualize trust, discuss validity issues, the advantages and disadvantages of their method and their personal experiences using the given technique. Several studies about trust have not covered these topics.

The main advantage of the book is that it provides solutions to many current challenges in trust research. It also outlines new directions for trust research by offering new methods and new directions for thinking about trust. The authors also highlight the fragmented nature of trust research, which must be overcome for the development of the field of research.

The conclusion repeated throughout the book is that there is a need for more work on trust. This handbook offers a platform of knowledge for those interested in trust, but also for those who are examining complex and multilevel phenomena similar to trust and those who are interested in relatively new and rarely-used techniques in the social sciences. 